In questo contributo vengono ricostruite le principali tappe del dibattito pubblico sul progetto per un museo nazionale sul fascismo, riepilogando in particolare le varie posizioni emerse sulla stampa e sul web.

This article focuses the main stages of the public debate on the project for a national museum of Fascism history, summarizing in particular the various positions which emerged in the press and on the web.

\title{
Alcune premesse
}

Predappio Nuova è la città di fondazione eretta dal fascismo negli anni Venti nel luogo di nascita di Mussolini, all'epoca Dovia, frazione di Predappio Vecchia (oggi Alta), sui colli forlivesi. Fra il 1934 e il 1937 viene edificata in paese anche una grande "Casa del fascio e dell'ospitalità", progettata da Arnaldo Fuzzi e inaugurata il $1^{\circ}$ maggio 1937.

Dopo la guerra Predappio, che sotto il regime era stata «Galilea di tutti noi», secondo le parole di Achille Starace, diventa ingombrante per la memoria pubblica nazionale; l'ormai ex Casa del fascio torna allo stato, che deve però accollarsi varie spese di recupero e quindi tenta vanamente di venderla. Nel 1954 il Comune la chiede come sede municipale, ma questo non viene consentito. Nel 1957 il presidente del consiglio democristiano Adone Zoli, anche lui di famiglia predappiese, si attiva per riportare in paese la salma di Mussolini, che viene tumulata 
nel cimitero di San Cassiano, dando il via a una prima stagione di pellegrinaggi nostalgici. Il sindaco comunista Egidio Proli afferma in quella circostanza: «Non ci ha fatto paura da vivo, non ci farà paura da morto».

Intanto la ex Casa del fascio viene destinata alla Pontificia opera di assistenza, che ne occupa un'ala con il suo laboratorio di falegnameria; l'edificio ospita anche alcuni uffici della Camera del lavoro, il circolo ricreativo social-comunista e vari esercizi privati. Nel 1983, in occasione del centenario della nascita di Mussolini, Predappio accoglie oltre 30.000 visitatori e ritorna al centro delle cronache. A metà anni Novanta, l'amministrazione (sempre di sinistra) di Ivo Marcelli concede la licenza ad alcuni negozi di gadget fascisti situati sulla via principale (viale Matteotti, già viale Mussolini). Nel 1999 lo stesso sindaco avvia il recupero della casa natale del duce, il "casone" sopra l'esedra del mercato, e nel 2000 organizza lì una grande mostra su di lui, facendone il primo passo di un ambizioso progetto di "museo urbano". Contestualmente viene avviato il recupero di alcuni altri edifici storici, come palazzo Varano (un tempo casa dei Mussolini, oggi sede del municipio), le Gallerie Caproni, la Rocca delle Caminate. Nel 2007 la ex Casa del fascio viene messa in sicurezza con un intervento da 300.000 euro; ma rimane inutilizzata; più tardi diventa "bene di interesse culturale" tutelato dalla soprintendenza. Intanto Predappio attira l'attenzione degli storici della memoria e dei cineasti: ne scrivono Massimo Baioni nel libro Luoghi di memoria curato da Mario Isnenghi e Patrizia Dogliani nel Dizionario del fascismo Einaudi; nel 2008 Marco Bertozzi realizza il documentario Predappio in Luce.

\section{Entra in scena Frassineti}

Nel maggio 2009 le lezioni amministrative vengono vinte, per un centinaio di voti, da Giorgio Frassineti, del Partito democratico. Geologo quarantacinquenne, Frassineti appare molto motivato ad affrontare il tema della memoria pubblica del paese (da consigliere comunale aveva tentato più volte di proibire i negozi di souvenir ideologici). Nel 2009 il neosindaco viene invitato a Braunau am Inn, la città natale di Hitler, per un convegno sulle patrie dei dittatori e le loro "memorie non volute". Da quella esperienza matura l'idea di combattere le nostalgie con una nuova strategia: quella della valorizzazione storica. Nel maggio 2011 il Comune entra infatti nel progetto Atrium, una iniziativa finanziata dal Consiglio d'Europa per la costruzione di un itinerario europeo sull' architettura totalitaria. Viene quindi allestita in città una mostra su Predappio città del Novecento. In quest'ambito maturano le prime idee di recupero dell'ex Casa del fascio. 
L'attivismo del nuovo sindaco trova grande riscontro nei media stranieri: alla fine del 2011 il "New York Times" ne scrive ripetutamente; e nel 2012 i giornalisti francesi Cyril Bérard e Samuel Picas, in collaborazione con "Le Monde", ne fanno il protagonista del web documentario La Duce vita. Nel 2013 l'Unione dei comuni della Romagna forlivese commissiona al Comune un Programma di riutilizzo e gestione, che viene redatto dal tecnico Carlo Giunchi e presentato in due parti (Analisi e Progetto) nel febbraio 2014. In questo documento si parla già della destinazione dell'edificio a polo culturale, comprendente un centro di documentazione e una museo permanente. Si allega uno studio storico sullo stabile effettuato da Ulisse Tramonti.

Intanto nel settembre 2013 viene inaugurata una grande mostra sul giovane Mussolini, che espone 200 dei circa 35.000 pezzi del collezionista locale Franco Moschi e si avvale della curatela di storici come Vittorio Emiliani e Maurizio Ridolfi. La mostra rimane aperta fino al maggio 2014, e attira l'attenzione dei giornali tedeschi e dell'“Indipendent". Nell'aprile 2014, in vista del rinnovo del mandato, Frassineti inserisce la questione ex Casa del fascio nel suo programma elettorale, il cui slogan è «Predappio bene comune».

A questo punto anche la stampa nazionale si accorge della questione. Il 19 "Il Giornale" lo intervista a firma di Luigi Mascheroni; poi chiama in causa diversi storici. Si dichiarano favorevoli Roberto Chiarini (che ritiene indispensabile elaborare, recuperandolo alla memoria democratica, anche il passato più ingombrante o traumatico, per evitare la rimozione e la nostalgia) e Nicholas Farrell. Contrari invece Francesco Perfetti (che denuncia il rischio di acuire le contrapposizioni politiche) e Luciano Canfora (che teme «un ibrido tra celebrazione e mummificazione»). Mario Cervi definisce l'idea «affascinante» per rivisitare l'identità nazionale, ma «inquietante» per il rischio di rinfocolare gli scontri ideologici.

Il 16 maggio Frassineti incontra il premier Matteo Renzi, che lo incoraggia a proseguire. Il 24 maggio il sindaco uscente viene confermato con un largo margine; e forte di questo risultato imprime al progetto una decisa accelerazione. In luglio il Comune sottopone il programma alla valutazione di un tavolo interistituzionale riunito dall'Assessorato alla cultura della Regione Emilia Romagna, del quale fanno parte, oltre alla Regione e al Comune stesso, il Comune di Forlì, la rete Insmli e l'Istituto di Forlì, il progetto Atrium, l'Anpi Emilia Romagna, la Fondazione Alfred Lewin (dedicata a un giovane ebreo socialista tedesco ucciso dai fascisti). 


\section{II ruolo di Marcello Flores}

In settembre proprio la Fondazione Lewin esplicita il proprio sostegno all'iniziativa. Il suo giornale "Una città" interpella Marcello Flores, che esplicita per la prima volta alcuni dei fondamenti del progetto: il valore critico della storia contro i pericoli della memoria, il carattere innovativo e originale del progetto, i suoi obiettivi formativi e divulgativi. In ottobre il Comune incarica quindi un gruppo di lavoro interdisciplinare, coordinato da Flores, di elaborarne le linee guida, a partire dal comparto espositivo. Ne fanno parte anche la museologa Patrizia Asproni e lo storico Giovanni Gozzini, insieme a Carlo Giunchi, Andrea e Vittorio Emiliani, Massimo Gardini e Patrizia Marti. A fine mese nell'ambito del neonato 900 fest, il festival europeo di storia del Novecento di Forlì, diretto da Flores, la cui prima edizione è dedicata alle dittature, viene presentato il programma di Giunchi.

Sempre su input di Flores, nel febbraio 2015 il Comune di Predappio aderisce ad Eurom, l'Osservatorio europeo della memoria con sede a Barcellona. L'8 giugno, il gruppo di lavoro licenzia le Linee guida, che vengono approvate il 18 giugno. A settembre il Consiglio comunale approva anche, con voto unanime, il Programma di valorizzazione, integrato nella parte progettuale con un capitolo inerente alla programmazione dell'intervento. Così parte l'iter per il trasferimento della proprietà dell'edificio dallo Stato al Comune.

A questo punto è "Il Foglio" a riproporre la questione all'opinione pubblica nazionale, intervistando Paolo Mieli (favorevole, come antidoto all'oblio e come sfida alla nostalgia) e Giovanni Sabbatucci (contrario invece per il rischio celebrativo e le relative ambiguità). La notizia esce anche su "Repubblica", "Espresso" e "Fatto quotidiano". Aldo Giannuli interviene sul suo blog rilevando lo scarso appeal del tema, che non tocca i veri problemi della contemporaneità. Cristina Portolano e Pietro Scarnera curano il webcomic Predappio città aperta. Intanto si riscontra un deciso aumento dell'afflusso a Predappio: i turisti dal 2014 al 2015 triplicano.

\section{II dibattito diventa nazionale}

Il 29 gennaio 2016 il sottosegretario alla Presidenza del Consiglio Luca Lotti si reca a Predappio per visionare la situazione e segnalare l'interessamento del governo; si parla di un possibile investimento di 2 milioni di euro; il mese dopo giungono anche i tecnici del Ministero per verificare le procedure. Contestualmente il deputato Pd Emanuele Fiano propone in Parlamento un disegno di legge contro l'ostentazione di simboli del fascismo che alimenta anche la discussione 
su Predappio (Frassineti è scettico, dichiarando di preferire l'arma della cultura a quella della proibizione; Fiano invece si schiererà a sostegno del suo progetto di riqualificazione). Il sindaco di Rimini, anch'egli del Pd, avvia una battaglia sulla sanzione della vendita di oggettistica fascista che porta a un provvedimento dell'Assemblea legislativa dell'Emilia Romagna, anch'essa accolta con scetticismo da Frassineti. A questo punto anche il "Museo di Predappio" diventa oggetto di un intenso dibattito, a più livelli.

Sul "Foglio" interviene direttamente il direttore Claudio Cerasa; i giornali di destra denunciano maliziosamente la posizione del governo, alimentando le critiche della sinistra. Il Pd risponde per bocca del deputato locale Marco Di Maio. È invece "La Stampa" a lanciare il dibattito tra gli storici. Il 17 febbraio pubblica uno scambio di opinioni tra Alessandro Campi (che deplora i rischi della demonizzazione) e Mario Isnenghi (convinto che i tempi siano maturi, ma dubbioso «sul come e il dove»), chiamati a Predappio a presentare le diverse edizioni del Giornale di guerra di Mussolini soldato. Interviene anche Miguel Gotor, storico ma anche senatore $\mathrm{Pd}$, che si dice favorevole, auspicando però un lavoro di alto profilo scientifico e «che non dimentichi i crimini del fascismo». Il 18 il quotidiano torinese pubblica in anteprima un documento di appoggio alle scelte del Comune di Predappio proposto da Flores e sottoscritto da 55 storici di 28 università o istituti di ricerca italiani e stranieri. Il testo insiste sulla serietà e il rigore scientifico del progetto, evoca diversi esempi esteri, ribadisce gli obbiettivi formativi. "Il manifesto" apre un dibattito: si schiera tendenzialmente per il no Davide Conti (secondo cui una struttura del genere a Predappio innescherebbe un «effetto paradossale»); favorevole invece Serge Noiret (che assegna la primogenitura dell'idea a un articolo di Marco Pizzo del 2012 e ricorda di averne già scritto nel 2013, in un articolo che rilevava la scarsa musealizzazione dei luoghi del fascismo e della Resistenza); decisamente contrario Enzo Collotti (che ritiene l'iniziativa «ingloriosa», se priva di un serio dibattito e non collocata nel quadro nazionale). Sugli "Stati generali" interviene invece favorevolmente David Bidussa, che parla di sfida culturale da vincere, per riconnotare i luoghi dalla memoria «pesante», attraverso il lavoro congiunto di più professionalità. Sul "Mulino" manifesta i suoi dubbi Luca Baldissara, che contesta in particolare il tenore del dibattito, schiacciato su un sì/no, oltretutto aprioristico, che poco avrebbe a che fare con la complessità della storia e molto più invece con la subalternità della cultura alla politica.

Ne parlano anche la Cnn e i giornali russi e giapponesi. Il 2 marzo 2016 l'ex Casa del fascio di Predappio diventa ufficialmente di proprietà comunale. Il dibattito arriva ormai ai quotidiani nazionali: intervengono Sergio Luzzatto (favorevole) e Carlo Ginzburg (contrario) sul domenicale del "Sole 24 Ore" (6 marzo). Ampie 
rassegne vengono pubblicate sulla rivista "Doppio Zero", a cura di Enrico Manera, dove intervengono tra gli altri Alberto Cavaglion (che teme il ripresentarsi di divisioni anacronistiche tra fascisti e antifascisti immaginari) e Simon Levis Sullam (che invece evidenzia la difficoltà di superare e neutralizzare il peso simbolico di certi spazi); e su "Una città", che avanza ora alcune critiche redazionali, chiamando poi in causa Mario Isnenghi, Anna Foa e ancora Cavaglion, tutti contrari; mentre favorevole è Giovanni Gozzini, che propone il metodo delle biografie esemplari e vede il museo come potenziale «laboratorio di intelligenza emotiva». A fine mese scrivono di Predappio anche Simonetta Fiori su "Repubblica" e Giovanni De Luna sulla "Stampa". Quest'ultimo denuncia soprattutto il rischio di mercificazione insito in un «un museo storico postmoderno che eclissa l'interpretazione ragionata della storia a vantaggio di un suo consumo usa e getta», e si mostra scettico sull'obbiettivo di una memoria comune che rifiuti scelte interpretative forti (sulla stessa linea anche Salvatore Lupo: «un museo del fascismo non può essere mai un'operazione neutra, né deve essere intesa come forma di riconciliazione»). Il 5 aprile "Internazionale" riporta un'analisi molto critica della scrittrice Igiaba Scego, che deplora la scelta di Predappio se si vuole fare davvero «un prodotto non manipolabile che spieghi e non glorifichi».

In aprile il Comune approva la ridefinizione del Progetto preliminare e inoltra alla Regione Emilia Romagna l'istanza per l'ottenimento, nell'ambito dei fondi strutturali europei, di un contributo di 2 milioni di euro, da aggiungersi ai 500.000 stanziati dalla Fondazione Carisp di Forlì e ai 500.000 di risorse proprie, per la realizzazione del I Lotto funzionale dell'edificio, contenente l'esposizione permanente. In maggio la Giunta avvia anche la procedura per l'Art Bonus, che consentirebbe l'erogazione di finanziamenti a beneficio del progetto con l'acquisizione del 65\% di credito di imposta. Intanto Flores replica alle critiche sia su "Doppio Zero" che su "Una città", pubblicando anche le Linee guida; Michele Smargiassi su "Repubblica Bologna" difende il genius loci dalla demonizzazione, rifiutando di fare di Predappio «una Chernobyl ideologica».

\section{Il coinvolgimento degli istituti storici della Resistenza}

A questo punto il Comune di Predappio propone alla rete nazionale degli istituti storici della Resistenza (all'epoca Insmli, oggi Istituto nazionale "Ferruccio Parri"), di seguire la progettazione scientifica dell'esposizione permanente. La proposta suscita un aspro dibattito interno e viene rigettata a maggioranza dal Cda il 13 maggio 2016, lasciando comunque facoltà ai singoli istituti della rete e ai singoli studiosi di rendersi disponibili. Contestualmente (il 9 maggio) esce un 
articolo di Serge Noiret che sostiene non solo la liceità, ma l'opportunità dell'operazione, e innesca una vasta discussione sui social, di cui riporta alcuni risultati anche su "PH Weekly".

La questione Predappio si incrocia in questa fase anche con l'apertura della mostra sul Culto del Duce al Musa di Salò, che inaugura il 29 maggio. A inizio luglio arriva anche da Londra una curiosa indicazione della locale sezione Anpi, che propone di intervenire sulla ex Casa del fascio modificandone la pianta e intersecandola con un «sentiero della resistenza».

A metà mese l'incarico della progettazione viene affidato ufficialmente dal Comune (stavolta solo a maggioranza) all'Istituto per la storia e le memorie del '900 - Parri Emilia-Romagna, con sede a Bologna e presieduto da Alberto De Bernardi, docente dell'Università di Bologna, che anche all'interno dell'Insmli aveva sostenuto l'opportunità dell'intervento. Il direttore dell'Istituto Luca Alessandrini rilascia alcune interviste, in cui rifiuta le ironie dei giornalisti sul museo del fascismo «fatto dai partigiani», e accetta invece esplicitamente la sfida di «decostruire» il fascismo, non attraverso le leggi repressive, bensì con una vasta e capillare operazione culturale. Gli altri istituti della rete emiliano-romagnola, informati ma non direttamente interpellati, rimangono in attesa di sviluppi; esce solo una nota di Laura Fontana e Daniele Susini, del coordinamento memoria di Rimini, che evidenzia alcune incognite del progetto.

Giulia Albanese, membro del Cda Insmli, risponde a Flores su "Politika", chiedendo un dibattito più largo e approfondito sulle modalità di storicizzazione e musealizzazione del fascismo. Il 3 settembre viene comunque sottoscritta la convenzione tra il Comune e il Parri, tra l'altro in occasione del conferimento al sindaco Frassineti del Premio dell'“Austrian Holocaust Memorial Award”, un'onoreficenza della comunità austriaca all'estero, per «il suo impegno nel cercare di creare un discorso obbiettivo sul fascismo attraverso un centro di documentazione del 900». Peraltro l'8 ottobre il sindaco partecipa a un dibattito nel teatro cittadino con la nipote di Himmler e l'8 novembre a Sanremo presenta il volume di memorie di Edda Negri Mussolini, nipote del duce, in una manifestazione organizzata dalla rete Lealtà e Azione.

A metà mese il Ministro Graziano Delrio è a Predappio per inaugurare la restaurata Rocca delle Caminate, divenuta un tecnopolo di interesse nazionale. Non mancano però le polemiche sull'idea di riaccendere il grande faro dell'edificio, costruito a suo tempo per segnalare a km di distanza la presenza di Mussolini nella sua residenza estiva. Tra gli interventi critici si segnalano quelli di Furio Colombo sul "Fatto Quotidiano"; disponibile alla discussione invece Mario Avagliano su Radio 3. 
A fine mese il Comune approva la costituzione del Comitato scientifico proposto dall'Istituto Parri per seguire la progettazione. È costituito, oltre che da Giunchi, Flores e De Bernardi, da Asproni e Gozzini, già coinvolti per le Linee guida; ci sono inoltre numerosi storici di vaglia (Roberto Balzani, Lorenzo Bertucelli, Gustavo Corni, Maurizio Ridolfi) e altri autorevoli professionisti del settore (il museologo Daniele Jalla e il massmediologo Enrico Menduni). Il comitato ha una proiezione internazionale, grazie alla presenza di Ruth Ben Ghiat, Patrick Bernhard, Marie-Anne Matard-Bonucci, Javier Rodrigo; più Serge Noiret, belga che insegna in Italia e Claudia Baldoli, italiana che insegna in Gran Bretagna.

A fianco del Comitato scientifico viene costituito anche un gruppo di lavoro operativo con Flores, De Bernardi, Gozzini, più Gardini e Marti (già membri del comitato consultivo nella fase precedente), l'architetto Andrea Milani e il saggista Carlo Greppi; la segreteria è affidata a Elena Bignami. La prima riunione del Comitato, coordinato dallo stesso Flores, si svolge a Predappio il 27 ottobre 2016. Contestualmente la SerInAr, l'ente di sostegno dell'Università a Forlì e Cesena, finanzia un programma di ricerca biennale denominato Predappio Europa, destinato a un'indagine sulla museografia europea sul tema dei totalitarismi. Coordinatore del programma è De Bernardi, che lo affida a Matteo Pasetti.

Intanto il 25 la Regione Emilia Romagna pubblica la graduatoria dei finanziamenti europei, garantendo al Comune di Predappio un milione di euro (una cifra considerevole, ma la metà di quanto richiesto). Viene inoltre sottoscritta una convenzione con la Fondazione della Cassa dei risparmi di Forlì per la gestione del fondo già stanziato dalla stessa.

Il 26 novembre Flores discute del progetto a Londra, nell'ambito della conferenza annuale Asmi, sottolineandone l'importanza per ovviare ai ritardi e alle carenze della memoria pubblica nazionale. Il 20 e 21 gennaio 2017 si svolge quindi a Predappio il Convegno internazionale Narrare il fascismo (di cui si riportano in questo spazio alcuni contenuti). In aprile prende poi il via il festival Forlì città del Novecento, promosso da Atrium, con appuntamenti importanti come il convegno Musei urbani del Novecento in Italia del 25 maggio e quello su Patrimonio dissonante e turismo sostenibile del 16 giugno.

\section{Il progetto dell'esposizione}

In aprile 1'Istituto Parri ER consegna al Comune di Predappio il progetto scientifico e museografico dell'esposizione permanente, intitolata L'Italia totalitaria. Stato e società in epoca fascista. Il progetto, approvato dal Comune, viene sottoposto alla valutazione dei rappresentanti istituzionali (Regione Emilia Romagna, 
Ministero dei beni e delle attività culturali e del turismo, Presidenza della Commissione cultura della Camera), che lo condividono nelle sue linee essenziali.

La stampa italiana sembra non accorgersene, mentre lo commentano Sabina Loriga su "The Conversation" e Fernando Devoto su "Future Pasts". Il 18 agosto il sindaco Frassineti fa visita al suo collega Mauro Robba, sindaco di Dongo e già promotore del Museo della fine della guerra inaugurato nel 2014. Il 28 settembre viene quindi bandito il concorso europeo per la progettazione, pubblicato sul sito dell'Unione dei Comuni (da cui viene tolto a inizio novembre, quando nasce il sito Progetto Predappio).

Il 5 ottobre Ruth Ben Ghiat, già membro del comitato scientifico, pubblica un articolo sul "New Yorker" sulla conservazione dei monumenti fascisti in cui esprime dubbi anche sull'operazione Predappio; l'articolo, prontamente ripreso da "Internazionale" il 27, suscita una ampia discussione critica. Intanto il 23 ottobre a Roma il progetto dell'esposizione permanente viene presentato pubblicamente alla stampa italiana ed estera. Il 9 dicembre il progetto viene presentato anche a Predappio; lo stesso giorno viene inaugurata una mostra che ne dettaglia le caratteristiche. Flores e De Bernardi ribadiscono la loro intenzione di «raccontare il carattere violento, illiberale, totalitario del fascismo fin dai suoi esordi» e l'obiettivo di «smontare documentando scientificamente i luoghi comuni che ancora esistono e si tramandano».

Intanto il collettivo Wu Ming pubblica sul suo blog Giap un dettagliato dossier sul tema in 3 puntate ( 27 ottobre, 7 novembre, 15 novembre). Il taglio è fortemente critico: le due fondamentali obiezioni sono che la proposta non tiene conto del peso simbolico della cornice, che risulta ben più forte delle velleità decostruttive dei proponenti; e che in quanto tale la proposta si configura come «un generatore automatico di cliché» corrivi al senso comune «post-antifascista», ritenuto dominante nella sinistra renziana, che sottovaluta le colpe del fascismo storico e minimizza i rischi di quello corrente. Quanto al merito del progetto, si definisce il documento del Parri «rabberciato e confuso». In particolare si rileva l'assenza di un quadro dettagliato dei contenuti e dei materiali da utilizzare; si contestano alcune scelte espositive, ritenute poco efficaci o troppo costose (ad esempio l'installazione morfica da 120.000 euro); si denunciano le lacune di contenuto, individuate nel fascismo di confine, nella riconquista della Libia, nella Rsi, nella mancata epurazione. Nel dossier ci sono poi vari inserti di approfondimento su questioni laterali, come i crimini del fascismo, la punibilità del neofascismo e la questione dei monumenti d'epoca, affrontati in modo serio, ma un po' slegati dal quadro di fondo. In una successiva intervista Wu Ming 1 ha sostenuto che «il mu- 
seo di Predappio è come Fico col fascismo al posto del cibo», cioè una proposta «spettacolarizzata, banalizzata, piena di non detti».

L'11 novembre 2017 esce anche un duro comunicato stampa firmato dall'Istituto di Forlì e da altre sigle locali, alcune delle quali avevano partecipato alla prima fase del progetto (ad es. la Fondazione Lewin e l'Anpi di Forlì-Cesena). Nel documento si contesta l'impronta dirigista dell'operazione, che non avrebbe in alcun modo coinvolto il territorio e avrebbe alla base una condotta non convincente e financo pericolosa; si denuncia la scarsa sensibilità per il contesto mostrata nel progetto; si rileva come il nuovo cronoprogramma sacrifichi la parte del centro di documentazione a tutto vantaggio dell'esposizione permanente.

Il 6 dicembre, in occasione dell'apertura della mostra, la neo presidente dell'Anpi Carla Nespolo esprime «ferma contrarietà» all'iniziativa per i rischi di celebrazione che implica e per il mancato coinvolgimentio delle associazioni reducistiche nella sua elaborazione. L'11 il sindaco e il presidente provinciale dell'Anpi Milo Gori si confrontano in diretta tv nello scenario della cripta Mussolini, suscitando anche le ire della nipote. Il 12 la deputata Lara Ricciatti, di Mdp, presenta una interrogazione sul tema alla Commissione cultura della Camera. Infine proprio il 29 dicembre esce sul "Corriere" un confronto serrato tra Paolo Pezzino, già docente dell'Università di Pisa, e Flores. Pezzino, tra i firmatari del manifesto del febbraio 2016, dice di aver cambiato idea e critica la sede predappiese, ritenuta poco significativa per il fascismo e rischiosa in termini di ordine pubblico. Flores ribadisce che l'intenzione dei promotori è quella di spiegare come la società italiana si sia adattata al fascismo; e di mettere i giovani a confronto con la storia, oltre le memorie.

\section{Perché sì}

Le motivazioni finora emerse a sostegno del Museo sono di diverso ordine. Il sindaco si è concentrato sull'esigenza di contendere gli spazi pubblici del paese alla memoria nostalgica e alla paccottiglia filofascista: «non celebrare, né sopportare, ma conoscere e raccontare» è un po' il mantra di Frassineti. In alcune interviste ha cercato di allargare il discorso alla necessità per l'Italia di elaborare il suo passato; e alla strada della cultura come via maestra per affrontare la crisi europea e il prevalere dei nazionalismi. Ma dietro la vocazione missionaria espressa dal sindaco, diversi critici hanno letto soprattutto una premura per il valore turistico, quindi commerciale, della proposta. Altri, come Wu Ming, hanno rilevato la differenza tra sostituire la nostalgia e affiancarla. Lorenzo Ferrari ("Il Post", 2 giu- 
gno 2016) ha scritto che «non si costruisce un museo nazionale perché un sindaco non sa che farsene di un edificio».

La Regione ha a sua volta sostenuto l'opportunità di affrontare di petto la difficile questione della memoria del fascismo, dicendosi intenzionata a costruire un progetto non celebrativo né solo conservativo, bensì culturale in senso lato e di portata europea. Anche qui non è mancato chi, come Giulia Albanese, ha osservato come la strada migliore per ottenere questi obiettivi non stia probabilmente nella operazione Predappio ma in una messa in rete dei numerosi luoghi di memoria emiliani.

Da parte del gruppo di storici che sostengono l'iniziativa, si è fatto leva soprattutto sulla contrapposizione tra la storia e la memoria, evidenziando come solo un'operazione culturale autorevole possa ovviare ai corti circuiti della rimozione e della nostalgia. In particolare Flores ha molto insistito sulla necessità di affrontare la storicizzazione del fascismo con un approccio scevro da pregiudiziali ideologiche e aperto ai nuovi linguaggi.

Un discorso a parte merita l'argomentazione incentrata sulla public history: narrare il fascismo in modo accurato e aggiornato al pubblico generico e non solo agli specialisti appare una sfida importante per la public history italiana, che recentemente ha costituito una sua associazione (l'Aiph - Associazione italiana di public history). Alla prima conferenza nazionale, svoltasi a Ravenna nel giugno 2017, un panel specifico è stato dedicato al museo di Predappio, con relazioni di Flores e De Bernardi; ma il caso è stato evocato anche in diversi panel internazionali. Noiret era già intervenuto sul tema al II congresso internazionale di Public History a Bogotà, nel luglio 2016, insistendo su una idea nuova di museo, senza confini fisici e con una forte proiezione nella rete. Con lui si sono schierati anche, in diverse sedi, Marcello Ravveduto ed Enrica Salvatori, tra i promotori della disciplina in Italia.

\section{Perché no: il perchè}

Più articolato il fronte del no, che ha visto sovrapporsi, talvolta anche in modo confuso, diversi ordini di obiezioni. Un primo livello di critiche riguarda la necessità stessa di istituire un museo del fascismo, rispetto ad altre priorità tematiche, ritenute più urgenti o adatte agli scopi educativi prefissi. $\mathrm{Ci}$ sono state così proposte per un museo del Novecento (Giannuli e Ferrari), dell'identità italiana (Perfetti), della Shoah, anch'esso più volte annunciato ma ancora inesistente (come rilevato ad esempio da Guido Crainz). Oppure, in chiave polemica, per un museo dei crimini del fascismo (Scego), dell'Antifascismo (Vacca) o del Neofascismo (Baldissara). 
C'è anche chi antepone al museo del fascismo la realizzazione di un museo della Resistenza, rilevandone l'assenza in Italia; a questi il sindaco e il suo entourage rispondono ricordando la rete di oltre 50 istituti storici sul tema (confondendoli però con musei). Alcuni hanno ricordato il progetto in corso presso la Casa della memoria di Milano, anche per rilevare il ruolo di direzione svolto anche in questa sede da Flores (Levis Sullam).

\section{Perché no: il quando (e il come)}

Un secondo ordine di problemi riguarda la scelta dei tempi, che alcuni ritengono comunque tardivi rispetto all'esigenza di storicizzare il fascismo (ad esempio Schwarz) e altri invece ancora prematuri (Canfora e Pennacchi). In particolare Collotti e Albanese hanno insistito sulla necessità di una elaborazione collettiva del passato fascista, paragonabile al percorso compiuto in Germania, come premessa indispensabile per qualunque discorso museale.

Wu Ming e altri hanno denunciato nell'operazione Predappio pericolose concessioni al discorso post-antifascista, individuando sia nelle posizioni del sindaco che in quelle di De Bernardi echi delle posizioni renziane. Non sono mancate anche critiche sul modo in cui è stata condotta l'operazione, con riferimenti in particolare alla conduzione verticistica e dirigistica del gruppo di lavoro, che non avrebbe aperto il confronto né a livello disciplinare né su quello territoriale, finendo poi per partorire una proposta «frettolosa e improvvisata».

A sinistra c'è anche chi ha parlato senza mezzi termini di spreco o almeno dubbia destinazione per un ammontare comunque considerevole di denaro pubblico. Peraltro la questione della gestione non è stata ancora esplicitata, anche sembra configurarsi l'ipotesi di una Fondazione ad hoc, a base mista. Del resto i fautori del museo hanno avuto buon gioco nel rilevare l'inopportunità di critiche pregiudiziali (Noiret) e i toni tribali della polemica innestata (Greppi).

\section{Perché no: il dove}

Un terzo e consistente ordine di obiezioni concerne la collocazione a Predappio, sia per motivi di principio che pragmatici. Dal primo punto di vista, si è fatto notare (ad esempio da Isnenghi) come ricondurre il fascismo a Predappio sarebbe una sineddoche pericolosa, che riproporrebbe un approccio storiografico superato, schiacciando il regime sul suo duce e finendo così per eludere proprio la spiegazione del suo consenso e dei suoi successi. 
Si è anche rilevato come Predappio sia un centro periferico e mal collegato; e come la presenza a fianco del futuro museo di luoghi come la casa natale o la tomba del duce rischino di compromettere le buone intenzioni degli organizzatori o addirittura di rovesciare gli effetti della struttura in un clamoroso boomerang celebrativo. Voci diverse come Isnenghi e Wu Ming (peraltro divise sul modo di storicizzare il fascismo) convengono sull'idea che in un progetto del genere la cornice rischi di prevalere; o almeno comporti «retrogusti e implicazioni di non facile gestione» e quindi da evitare, per non creare polemiche inutili e non sprecare i soldi disponibili.

Rispetto alla collocazione a Predappio sono state proposte tre fondamentali alternative:

1) la maggior parte degli interlocutori ha sostenuto che un museo nazionale del fascismo debba trovare collocazione in una grande città, restringendo fondamentalmente il campo a Roma (capitale del paese, sede del governo mussoliniano, tempio dell'architettura fascista - in diversi hanno indicato l'Eur) o tutt'al più a Milano (luogo di nascita e di morte del movimento, ma anche capitale della Resistenza);

2) alcuni, evidenziando la necessità di mostrare soprattutto il volto criminale del regime, hanno suggerito di collocarlo in un luogo significativo in questo senso (ad esempio Levis Sullam ha proposto Fossoli);

3) altri hanno sottolineato come tanto per la natura del paese che per quella del fascismo, l'operazione più efficace sarebbe la spiegazione, valorizzazione ed eventualmente riconnotazione dei tanti luoghi della memoria del fascismo ancora presenti e visibili sul territorio nazionale, magari attraverso la costruzione di un "museo diffuso" (come suggerisce anche Eraldo Affinati nel suo Camminare per l'Italia fascista).

L'opportunità di lavorare su Predappio non è stata in questo senso scartata in assoluto; ma si è proposto di dedicare eventualmente lì un museo a Mussolini (proposta su cui concordano storici molto diversi come Cardini, Barberis, Baldissara, Spagnolo); o di inserire la cittadina forlivese in una rete di luoghi significativi per la vicenda fascista. A questo proposito si è fatto notare che proprio in Romagna si snoda il «museo diffuso dell'abbandono» In loco, promosso dall'associazione spazi indecisi, che ha tra l'altro due itinerari dedicati propri all'architettura fascista. Peraltro i fautori del museo, riconoscendo che altre collocazioni sarebbero probabilmente più opportune a tavolino, hanno fatto notare come in 70 anni esse non si siano mai concretizzate e anzi non abbiano avuto nessuna reale possibilità di farlo. 


\section{Perché no: il cosa}

Infine, posta l'idea di un progetto già in corso su Predappio, ci sono state delle obiezioni sulle finalità della nuova eventuale struttura. L'approccio museale classico è stato rigettato perché ritenuto a rischio di celebrazione (anche se questa argomentazione è apparsa a molti, tra cui Noiret, viziata da una idea superficiale e anacronistica di "museo").

È vero peraltro che gli stessi promotori hanno più volte oscillato nell'uso dei termini, parlando alternativamente (e spesso a seconda dell'interlocutore e delle circostanze) di spazio museale, spazio espositivo, centro di interpretazione, centro di documentazione, centro culturale. Dal progetto sembra di poter dedurre che si intende realizzare un polo culturale complesso, comprendente almeno due elementi distinti: uno spazio espositivo permanente (che dovrebbe occupare l'ala nord); e un centro di documentazione (a cui sarebbe destinata invece l'ala ovest, che ospiterebbe anche, negli altri piani, gli uffici e i servizi al pubblico). Alcuni hanno sottolineato l'opportunità di insistere piuttosto con le esposizioni temporanee (Sabbatucci); altri propendono invece per centro di conservazione e ricerca (Canfora, ad esempio, propone una grande biblioteca tematica).

Per quanto riguarda il contenuto, si è parlato via via di fascismo, di Italia fascista, dell'Italia nel ventennio fascista, di prima metà del Novecento, di totalitarismi. Da ultimo il progetto utilizza la dizione «Italia totalitaria, stato e società in epoca fascista».

Interessante anche il confronto con il dibattito internazionale: sia i promotori che $\mathrm{i}$ contrari infatti hanno tirato in ballo il quadro europeo e in particolare il caso tedesco. Gli uni per indicare l'esempio di Monaco come modello di luogo di memoria del terrore totalitario che è stato utilmente trasformato in un centro di studio e di formazione (e a questo proposito sono stati ricordati anche i numerosi musei della Shoah, che certo non hanno intenti celebrativi). Gli altri, concentrando piuttosto l'attenzione su Berlino, per rilevare come la musealizzazione dei regimi totalitari vada pensata in modo sobrio ed aperto, dal basso, togliendo piuttosto che aggiungendo e creando nello spazio una sorta di "ipertesto della memoria".

\section{Risorse online}

Progetto Predappio

https://progettopredappio.it/

La Duce Vita. Un documentario interattivo, di Cyril Bérard e Samuel Picas http://www.lemonde.fr/laducevita/\#/prehome 